\title{
Method of Lines for Third Order Partial Differential Equations
}

\author{
Mustafa Kudu, Ilhame Amirali \\ ${ }^{1}$ Department of Mathematics, Faculty of Art and Science, Erzincan University, Erzincan, Turkey \\ ${ }^{2}$ Department of Mathematics, Faculty of Art and Science, Sinop University, Sinop, Turkey \\ Email: muskud28@yahoo.com, ailhame@gmail.com
}

Received December 5, 2013; revised January 5, 2014; accepted January 10, 2014

Copyright (C) 2014 Mustafa Kudu, Ilhame Amirali. This is an open access article distributed under the Creative Commons Attribution License, which permits unrestricted use, distribution, and reproduction in any medium, provided the original work is properly cited. In accordance of the Creative Commons Attribution License all Copyrights (c) 2014 are reserved for SCIRP and the owner of the intellectual property Mustafa Kudu, Ilhame Amirali. All Copyright (C) 2014 are guarded by law and by SCIRP as a guardian.

\section{ABSTRACT}

The method of lines is applied to the boundary-value problem for third order partial differential equation. Explicit expression and order of convergence for the approximate solution are obtained.

\section{KEYWORDS}

\section{Method of Lines; Partial Differential Equation; Convergence; Error Estimates}

\section{Introduction}

We consider the boundary value problem for the third order differential equation in the domain $\Omega\{0<x<p, 0<y<q\}$ :

$$
\begin{gathered}
\frac{\partial^{3} u}{\partial x^{3}}+\frac{\partial^{3} u}{\partial x \partial y^{2}}=f(x, y), \\
u(x, 0)=\psi_{1}(x), u(x, q)=\psi_{2}(x), \\
u(0, y)=g_{1}(y), u(p, y)=g_{2}(y), \\
\frac{\partial u}{\partial x}(0, y)=g_{3}(y),
\end{gathered}
$$

where $\psi_{1}(x), \psi_{2}(x), g_{1}(y), g_{2}(y), g_{3}(y)$ are sufficiently smooth functions.

The problems of type (1)-(4) arise in many mathematical and scientific applications [1-3]. In this study, we construct first order accurate differential difference scheme for this problem and give error estimate for its solutions. The approach to the construction of the discrete problem and the error analysis for the approximate solution are similar to those in [4].

Let the solution of the problem (1)-(4) have a bounded derivative $\frac{\partial^{7} u}{\partial x \partial y^{6}}$ in the domain $\bar{\Omega}\{0 \leq x \leq p, 0 \leq y \leq q\}$.

\section{Differential-Difference Algorithm and Convergence}

We divide the domain $\Omega$ into $n+1$ stripe by lines $y=y_{k}=k h\left(k=1,2, \cdots, n ; h=\frac{q}{n+1}\right)$. On this lines the problem (1)-(4) we approximate by the following differential difference problem: 


$$
\begin{gathered}
U_{k+1}^{\prime \prime \prime}+10 U_{k}^{\prime \prime \prime}+U_{k-1}^{\prime \prime \prime}+\frac{12}{h^{2}}\left(U_{k+1}^{\prime}-2 U_{k}^{\prime}+U_{k-1}^{\prime}\right)=f_{k+1}(x)+10 f_{k}(x)+f_{k-1}(x), \\
U_{0}(x)=\psi_{1}(x), U_{n+1}(x)=\psi_{2}(x), \\
U_{k}(0)=g_{1}\left(y_{k}\right), U_{k}(p)=g_{2}\left(y_{k}\right), \\
U_{k}(0)=g_{3}\left(y_{k}\right) .
\end{gathered}
$$

Let we rewrite the problem (5)-(8) in the form

$$
\begin{gathered}
A U^{\prime \prime \prime}-\frac{12}{h^{2}} M U^{\prime}=F(x), \\
U(0)=g_{1}^{(0)}, U(p)=g_{2}^{(p)}, \quad U^{\prime}(0)=g_{3}^{(0)},
\end{gathered}
$$

where

$$
M=\left(\begin{array}{cccccc}
2 & -1 & 0 & \cdots & 0 & 0 \\
-1 & 2 & -1 & \cdots & 0 & 0 \\
0 & -1 & 2 & \cdots & 0 & 0 \\
\vdots & \vdots & \vdots & \ddots & \vdots & \vdots \\
0 & 0 & 0 & \cdots & 2 & -1 \\
0 & 0 & 0 & \cdots & -1 & 2
\end{array}\right), A \equiv 12 I-M=\left(U_{1}(x), U_{2}(x), \cdots, U_{n}(x)\right), \quad\left(\begin{array}{cccccc}
10 & 1 & 0 & \cdots & 0 & 0 \\
1 & 10 & 1 & \cdots & 0 & 0 \\
0 & 1 & 10 & \cdots & 0 & 0 \\
\vdots & \vdots & \vdots & \ddots & \vdots & \vdots \\
0 & 0 & 0 & \cdots & 10 & 1 \\
0 & 0 & 0 & \cdots & 1 & 10
\end{array}\right),
$$

I-unit matrix,

$$
\begin{aligned}
& F(x)=\left(F_{1}(x), F_{2}(x), \cdots, F_{n}(x)\right), F_{1}(x)=f\left(x, y_{2}\right)+10 f\left(x, y_{1}\right)+f(x, 0)-\psi_{1}^{\prime \prime \prime}(x)-\frac{12}{h^{2}} \psi_{1}^{\prime}(x), \\
& F_{k}(x)=f\left(x, y_{k+1}\right)+10 f\left(x, y_{k}\right)+f\left(x, y_{k-1}\right), k=2,3, \ldots, n-1, \\
& F_{n}(x)=f(x, q)+10 f\left(x, y_{n}\right)+f\left(x, y_{n-1}\right)-\psi_{2}^{\prime \prime \prime}(x)-\frac{12}{h^{2}} \psi_{2}^{\prime}(x), \\
& g_{1}^{(0)}=\left(g_{1}\left(y_{1}\right), g_{1}\left(y_{2}\right), \cdots, g_{1}\left(y_{n}\right)\right), g_{2}^{(p)}=\left(g_{2}\left(y_{1}\right), g_{2}\left(y_{2}\right), \cdots, g_{2}\left(y_{n}\right)\right), g_{3}^{(0)}=\left(g_{3}\left(y_{1}\right), g_{3}\left(y_{2}\right), \cdots, g_{3}\left(y_{n}\right)\right) .
\end{aligned}
$$

The matrix $M$ can be diagonalized as [5,6]

$$
M=B^{-1} \operatorname{diagonal}\left(\lambda_{1}, \lambda_{2}, \cdots, \lambda_{n}\right) B,
$$

with

$$
B=B^{-1}=\left(b_{k s}\right)_{k, s=1}^{n}=\left((-1)^{k+s} \sqrt{\frac{2}{n+1}} \sin \frac{\pi k s}{n+1}\right)_{k, s=1}^{n}, \quad \lambda_{s}=\frac{4}{h^{2}} \cos ^{2}\left(\frac{\pi s}{2(n+1)}\right), s=1,2, \cdots, n .
$$

Multiplying Equation (9) on the left by $B$ we have

$$
\begin{gathered}
\left(12-\lambda_{s}\right) \varphi_{s}^{\prime \prime \prime}-\frac{12}{h^{2}} \lambda_{s} \varphi_{s}^{\prime}=l_{s}(x), \\
\varphi_{s}(0)=\varphi_{s 0}, \varphi_{s}(p)=\varphi_{s p} \\
\varphi_{s}^{\prime}(0)=\varphi_{s 0}^{\prime}, s=1,2, \cdots, n
\end{gathered}
$$

where

$$
\varphi_{s}(x)=\sum_{k=1}^{n} b_{s k} U_{k}(x), l_{s}(x)=\sum_{k=1}^{n} b_{s k} F_{k}(x), \quad \varphi_{s 0}=\sum_{k=1}^{n} b_{s k} g_{1}\left(y_{k}\right), \varphi_{s p}=\sum_{k=1}^{n} b_{s k} g_{2}\left(y_{k}\right),
$$




$$
\varphi_{s 0}^{\prime}=\sum_{k=1}^{n} b_{s k} g_{3}\left(y_{k}\right), s=1,2, \cdots, n \text {. }
$$

The solution of (10)-(12) containing the third order ordinary differential equation with constant coefficients can be explicitly found

$$
\begin{aligned}
\varphi_{s}(x)= & -\int_{0}^{p} G_{s}(x, \xi) \bar{l}_{s}(\xi) \mathrm{d} \xi+\frac{\sinh \sigma_{s} p-\sinh \sigma_{s}(p-x)-\sinh \sigma_{s} x}{\sigma_{s}\left(\cosh \sigma_{s} p-1\right)} \\
& {\left[\varphi_{s 0}^{\prime}-\frac{1}{\sinh \sigma_{s} p} \int_{0}^{p} \sinh \sigma_{s}(p-\xi) \bar{l}_{s}(\xi) \mathrm{d} \xi\right]+\frac{\cosh \sigma_{s} x-1}{\cosh \sigma_{s} p-1}+\frac{\cosh \sigma_{s} p-\cosh \sigma_{s} x}{\cosh \sigma_{s} p-1} \varphi_{s 0} }
\end{aligned}
$$

where

$$
G_{s}(x, \xi)= \begin{cases}\frac{\sinh \sigma_{s} x \cdot \sinh \sigma_{s}(p-\xi)}{\sigma_{s} \sinh \sigma_{s} p}, & x \leq \xi \\ \frac{\sinh \sigma_{s}(p-x) \cdot \sinh \sigma_{s} \xi}{\sigma_{s} \sinh \sigma_{s} p}, & x \geq \xi .\end{cases}
$$

Therefore the solution of (5)-(8) can be expressed as

$$
\begin{aligned}
& U_{k}(x)=\sum_{s=1}^{n}(-1) \sqrt{\frac{k}{n+s}} \sin \frac{\pi k s}{n+1} \cdot\left\{-\int_{0}^{p} G_{s}(x, \xi) \bar{l}_{s}(\xi) \mathrm{d} \xi+\frac{\sinh \sigma_{s} p-\sinh \sigma_{s}(p-x)-\sinh \sigma_{s} x}{\sigma_{s}\left(\cosh \sigma_{s} p-1\right)}\right. \\
& \left.\cdot\left[\varphi_{s 0}^{\prime}-\frac{1}{\sinh \sigma_{s} p} \int_{0}^{p} \sinh \sigma_{s}(p-\xi) \bar{l}_{s}(\xi) \mathrm{d} \xi\right]+\frac{\cosh \sigma_{s} p-\cosh \sigma_{s} x}{\cosh \sigma_{s} p-1} \varphi_{s 0}+\frac{\cosh \sigma_{s} x-1}{\cosh \sigma_{s} p-1} \varphi_{s p}\right\},
\end{aligned}
$$

where

$$
\bar{l}_{s}(x)=\frac{1}{12-\lambda_{s}} \int_{0}^{x} l_{s}(t) \mathrm{d} t .
$$

Now we investigate the error of the approximate solution. For the error $z_{k}(x)=u\left(x, y_{k}\right)-U_{k}(x)$ we have the following boundary value problem:

$$
\begin{aligned}
& z_{k+1}^{\prime \prime \prime}+10 z_{k}^{\prime \prime \prime}+z_{k-1}^{\prime \prime \prime}+\frac{12}{h^{2}} \cdot\left(z_{k+1}^{\prime}-2 z_{k}^{\prime}+z_{k-1}^{\prime}\right)=R_{k}(x), \\
& z_{0}(x) \equiv 0, z_{k+1}(x) \equiv 0, \quad z_{k}(0) \equiv 0, z_{k}(p) \equiv 0, \quad z_{k}^{\prime}(0)=0, k=1,2, \cdots, n,
\end{aligned}
$$

where

$$
\begin{gathered}
R_{k}(x)=-\frac{h^{4}}{20} \cdot \frac{\partial^{7} u}{\partial x \partial y^{6}}\left(x, \bar{y}_{k}\right),\left|R_{k}(x)\right| \leq C_{1} h^{4}, C_{1}=\text { const }, k=1,2, \cdots, n, y_{k-1}<\bar{y}_{k}<y_{k+1} . \\
\left(12-\lambda_{s}\right) \varphi_{s}^{\prime \prime \prime}-\frac{12}{h^{2}} \lambda_{s} \varphi_{s}^{\prime}=l_{s}(x), \quad \varphi_{s}(0)=0, \varphi_{s}(p)=0, \varphi_{s}^{\prime}(0)=0
\end{gathered}
$$

or

$$
\varphi_{s}^{\prime \prime}-\sigma_{s}^{2} \varphi_{s}=\bar{l}_{s}(x)+K_{s}, \quad \varphi_{s}(0)=0, \varphi_{s}(p)=0, \varphi_{s}^{\prime}(0)=0, \quad \varphi_{s}(x)=\sum_{k=1}^{n} b_{s k} \gamma_{k}(x), \bar{l}_{s}(x)=\int_{0}^{x}\left(\sum_{k=1}^{n} b_{s k} R_{k}(t)\right) \mathrm{d} t .
$$

Next for

$$
K_{s}=-\frac{\int_{0}^{p} \sinh \sigma_{s}(p-\xi) \bar{l}_{s}(\xi) \mathrm{d} \xi}{\int_{0}^{p} \sinh \sigma_{s}(p-\xi) \mathrm{d} \xi},\left(\sinh \sigma_{s}(p-x)>0\right) .
$$

By the mean value theorem we have 


$$
K_{s}=-\bar{l}_{s}(\eta), 0<\eta<p .
$$

Then

$$
\bar{l}_{s}(x)+K_{s}=\bar{l}_{s}(x)-\bar{l}_{s}(\eta)=\bar{l}_{s}^{\prime}(\xi)(x-\eta)=l_{s}(\xi)(x-\eta) .
$$

Since $l_{s}(x)=\sum_{k=1}^{n} b_{s k} R_{k}(x)$, then it follows that

$$
\left|\bar{l}_{s}(x)+K_{s}\right| \leq\left|l_{s}(\xi)\right| p \leq \max _{0 \leq x \leq p} l_{s}(x) \mid p \leq C_{1} p \sqrt{2 q} h^{3.5} .
$$

Further, we note that $\int_{0}^{p} G(x, \xi) \mathrm{d} \xi \leq \frac{1}{\sigma_{s}^{2}}$ and

$$
\left|\varphi_{s}(x)\right|=\left|\int_{0}^{p} G_{s}(x, \xi)\left[\bar{l}_{s}(\xi)+K_{s}\right] \mathrm{d} \xi\right| \leq \frac{1}{\sigma_{s}^{2}} \max _{0 \leq x \leq p}\left|\bar{l}_{s}(x)+K_{s}\right| \leq \frac{C_{1} p \sqrt{2 q} h^{3.5}}{\sigma_{s}^{2}\left(12-\lambda_{s}\right)}=\frac{C_{1} p \sqrt{q} h^{5.5}}{24 \sqrt{2} \cos ^{2} \frac{\pi s}{2(n+1)}}, s=1,2, \cdots, n .
$$

Hence

$$
\left|z_{k}(x)\right| \leq \sum_{s=1}^{n}\left|b_{k s}\right|\left|\varphi_{s}(x)\right| \leq \sqrt{\frac{2}{n+1}} \cdot \frac{C_{1} p \sqrt{q}}{24 \sqrt{2}} h^{5.5} \sum_{s=1}^{n} \frac{1}{\cos ^{2} \frac{\pi s}{2(n+1)}}
$$

Using here the inequality $\sin x>\frac{2}{\pi} x\left(0<x<\frac{\pi}{2}\right)$, and taking into account $\sum_{k=1}^{n} \frac{1}{k^{2}}<\sum_{k=1}^{\infty} \frac{1}{k^{2}}=\frac{\pi^{2}}{6}$

it follows that

$$
\left|z_{k}(x)\right| \leq \frac{C_{1} p h^{6}}{24}(n+1)^{2} \sum_{s=1}^{n} \frac{1}{(n+1-s)^{2}} \leq \frac{C_{1} \pi^{2} p q^{2}}{144} h^{4},
$$

i.e., fourth order convergence for the approximate solution is established.

\section{REFERENCES}

[1] A. I. Kozhanov, "Mixed Boundary Value Problem for Some Classes of Third Order Differential Equations,” Matematicheskii Sbornik, Vol. 118 (160), No. 4 (8), 1982, pp. 504-522. (in Russian)

[2] A. I. Kozhanov, “Mixed Problem for One Class of Quasilinear Equation of Third Order,” In: Boundary Value Problems for Nonlinear Equations, Novosibirsk, 1982, pp. 118-128. (in Russian)

[3] S. A. Gabov and A. G. Sveshnikov, "Problems of the Dynamics of Stratified Fluids,” Nauka, Moscow, 1986, p. 288. (in Russian)

[4] G. M. Amiraliyev and P. Okcu, "Error Estimates for Differential Difference Schemes to Pseudo-Parabolic Initial-Boundary Value Problem with Delay,” Computers \& Mathematics with Applications, Vol. 18, No. 3, 2013, pp. $283-292$.

[5] S. B. Nemchinov, “On the Finite Difference Method to the Elliptic Boundary Value Problems,” Journal of Computational and Applied Mathematics, Vol. 2, 1962, pp. 418-436. (in Russian)

[6] A. A. Samarskii, “The Theory of Difference Schemes,” Marcel Dekker, New York, 2001. 